\title{
A TWO-DIMENSIONAL WHITE-LIGHT AMPLITUDE INTERFEROMETER
}

James B. Breckinridge

Jet Propulsion Laboratory (CALTECH), Pasadena, CA., 91103*

\section{ABSTRACT}

A review of the applications of a point-symmetric, 180-degree, rotational shearing interferometer is given. Material includes, photographs of the Michelson stellar interferometer fringes from $\alpha$ Lyr, measures of amplitude of phase excursions in the atmosphere, and a measure of the order of interference in speckle patterns. New material includes unpublished double star speckle patterns to illustrate the isoplanatic patch, a measure of the time fluctuations of the earths' atmosphere, and measurements of the properties of a 256 channel linear reticon.

\section{INTRODUCTION}

Construction of a field-folding interferometer to observe the Michelson stellar interferometer fringes at large telescopes was proposed in $1972^{1}$ The interferometer was completed in $1974^{2}$. This paper summarizes observations recorded with the instrument between 1974 and the summer of $1976^{3}$. That work was done realizing that the instrument provides a display of the modulus of the mutual coherence function. Additional theoretical work has shown that it may be practical to consider this technique for near diffraction limited image reconstruction of images blurred by atmospheric turbulence ${ }^{4}$

An interferometer similar to the one discussed here, but with a variable angle of rotational shear has been developed and used by $C$. Roddier ${ }^{5}$.

\section{THE INSTRUMENT}

Figure 1 shows the most critical and difficult to fabricate part of the interferometer. The telescope pupil is relayed through the crossed roofs of the interferometer. The pupil is vlewed looking back from the right. The principles of operation are best understood if we Imagine a star positioned 


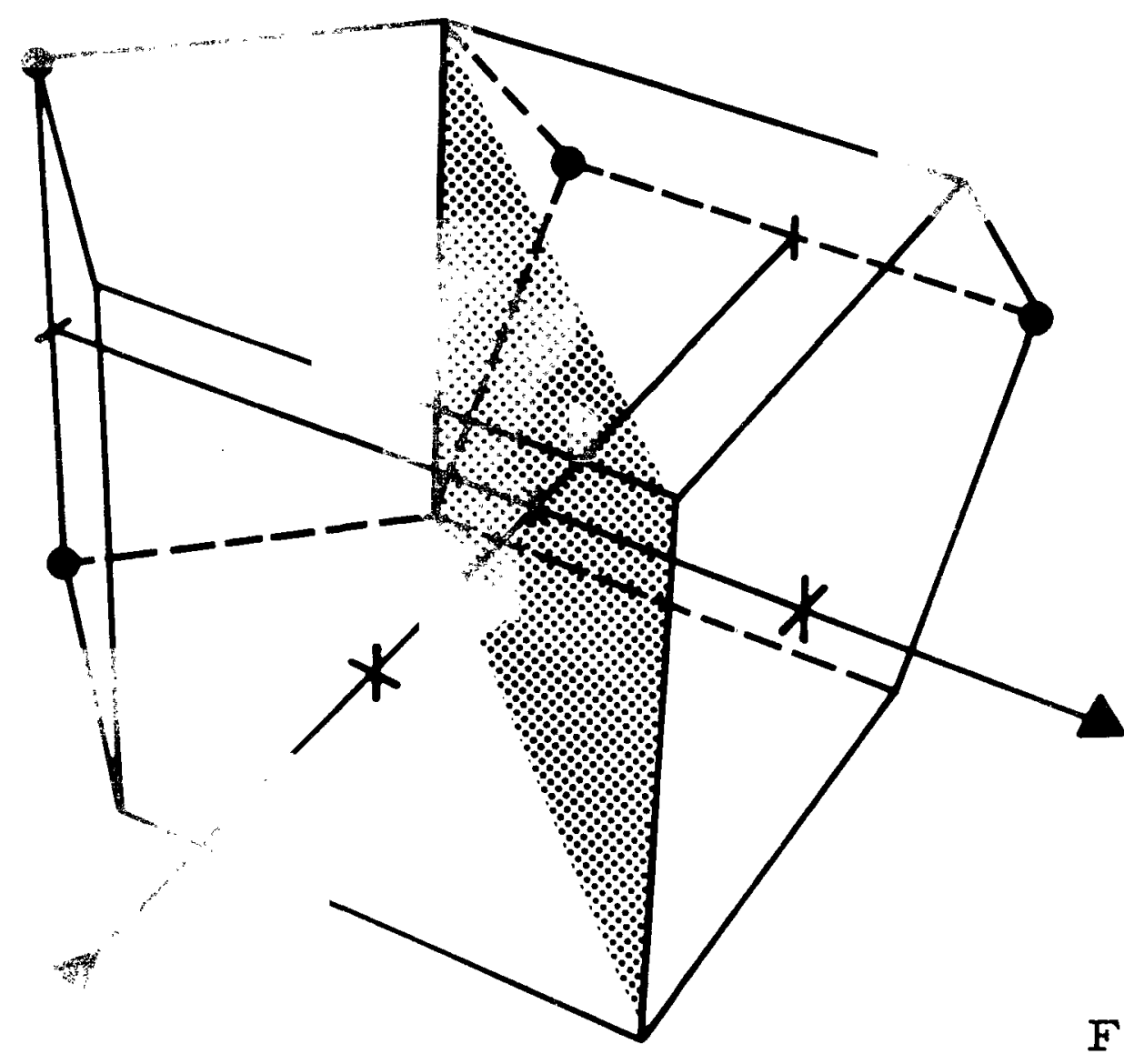

FIGURE 1

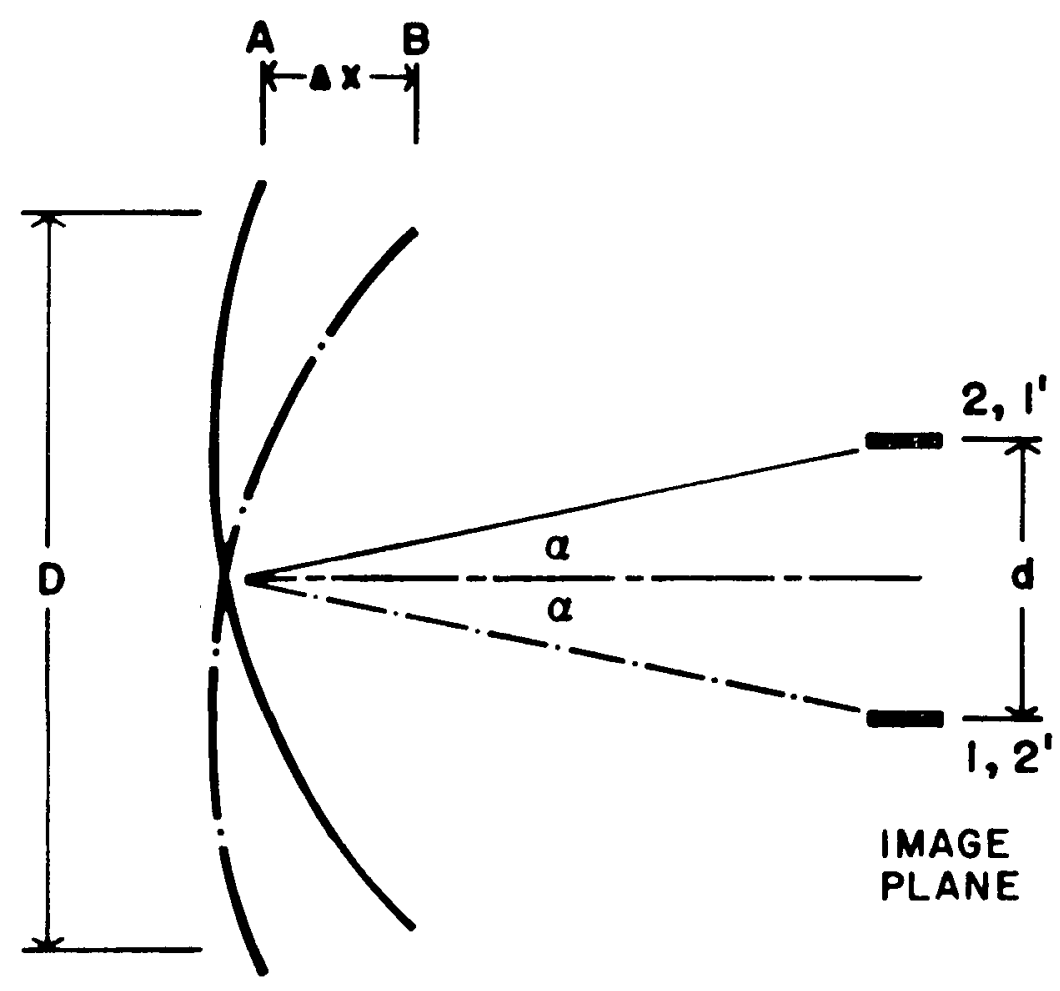

$$
\left.\underset{B^{\prime}}{k} \Delta x \rightarrow\right|_{A^{\prime}}
$$

PUPIL

PLANE

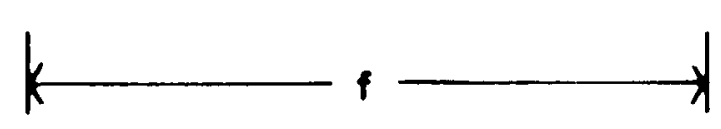

FIGURE 2 
slightly off the system axis. The light from the pupil relayed into the prisms is then a slightly tilted plane wave. At the output, because of the field folding, or rotational shear of the interferometer, two plane waves appear, tilted with respect to each other. The optical path difference in one arm of the interferometer is precisely equal to that in the other arm, the two plane waves are coherent with each other, they interfere and fringes are visible over the pupil image - even in white light.

\section{PHOTOGRAPH OF MICHELSON STELLAR FRINGES}

A photograph of the Michelson stellar interferometer fringes was recorded in quasi-monochromatic 1ight. It has been published ${ }^{2}$ Copies of the photographs were available at the meeting of Colloquium No. 50. The fringes were recorded for $\alpha$ Lyr at the 1.5 meter McMath Solar Telescope of the Kitt Peak National Observatory Fringe visibility is high across the pupil, to the edge, verifying that the apparent angular diameter of $\alpha$ Lyr $15<0.03$ arc-seconds.

\section{AMPLITUDE OF PHASE EXCURSIONS}

A visual measurement of the peak-to-peak amplitude of phase excursions in the earth's atmosphere was made in the "white light" from several stars. This information appears in the literature 3,6 . The measured peak-to-peak total physical motion required for active (or adaptive) optics for pupil points separated by 1.5 meters is about 2 microns. It was verified that the fringe amplitude in waves goes as the $5 / 6$ th power of the pupil point separation.

\section{INTERFERENCE PHENOMENA IN STELLAR SPECKLE}

Using the information on the amplitude of the phase excursions, the number of individual speckles in a stellar speckle pattern and the physical appearance of a stellar speckle pattern recorded through a special "two spectrum window" optical filter our understanding of the formation of stellar speckles has improved. This material has been published and further details will not be given here ${ }^{7}$. 


\section{ILLUSTRATIONS OF THE ISOPLANATIC PATCH}

The Kitt Peak Speckle Camera was designed to have a large number of pixel elements so that a relatively large field of view of stellar speckles could be recorded. A description of this camera, with photographs of the stellar speckle pattern from double stars is in press now 8 . Copies of the photographs were available at the meeting of Colloquium No. 50 .

\section{WAVELENGTH DEPENDENCE OF THE FIELD-OF-VIEW}

This 180-degree rotational shearing interferometer was used in preliminary experiments to try and reconstruct a diffraction limited image of the solar limb $^{3}$ : The field-of-view which can be processed through the interferometer prisms depends upon the temporal bandwidth. Two other groups working independently of each other have devised schemes to "achromatise" amplitude interferometers $^{9,10}$. For the experiments performed on the sun, time did not permit construction of an achromatizer. Field-of-view, and band-width were adjusted.

Figure 2 shows the parameters for the calculation of optical filter bandwidth. At the image plane of diameter $d$, a distance $f$ from the pupil we observe the superposition of two pupils of diameter D, rotationally sheared by 180 degrees about a point at the intersection of the optical axis and the pupil surface. $A$ and $A^{\prime}$ are points at the edge of the pupil $A A^{\prime}$. $B$ and $B^{\prime}$ are points at the edge of the pupil $\mathrm{BB}^{\prime}$ We assume that the field angle $\alpha$, is given by $\alpha=d /(2 f)$. Below the axis we see that a wavefront leaving point $B^{\prime}$ arrives at point 2 at time $C \cdot \Delta \mathrm{X}$ after that wavefront which left point $A$. The constant $\mathrm{C}$ is the speed of light. To minimize chromatic effects we require a narrow band optical filter which will yield a coherence length at least as long as $2 \Delta \mathrm{X}$.

In Figure 2 we show the two apparent pupils as viewed in the space of the detector plane; one indicated by $A^{\prime} A$ is turned around with respect to the other indicated by $B^{\prime} B$. Both are images of the same pupil. We wish to view fringes across the combined pupil areas, from the right, looking back to the left, through the aperture of diameter $d$, located at the image plane. Below the axis we see that a ray from $A^{\prime}$ to the superposed image plane point 2 is shorter by $\Delta \mathrm{x}$ than the ray from points $\mathrm{B}^{\prime}$ to the same superposed image plane point 2 . 
To minimize fringe apodization by chromatic effects we require a narrow band optical filter which will yield a coherence length at least as long as $2 \Delta x$. From the geometry given in the figure, we can write:

$$
\alpha=\frac{\Delta \mathrm{x}}{(\mathrm{n} / 2)}=\frac{2 \mathrm{c} \Delta \mathrm{T}}{\mathrm{D}}
$$

where $\Delta T$ is the light time transit from point $B^{\prime}$ to $A^{\prime}$, and also that for transit from point $A$ to $B$.

We rewrite the above equation to give

$$
\Delta T=\left(\frac{D}{2}\right)\left(\frac{\alpha}{C}\right),
$$

and use the condition relating time and bandwidth to give a correlated wavepacket

$$
\Delta T \quad \Delta v=\frac{1}{2 \pi},
$$

and use the we11 known relationship,

$$
\Delta \lambda=\bar{\lambda} \frac{\Delta \nu}{\bar{v}}
$$

where $\bar{\lambda}$ is the mean wavelength and $\bar{v}$ is the mean frequency, to obtain

$$
\Delta \lambda=\bar{\lambda}^{2}\left(\frac{1}{\pi}\right)\left(\frac{1}{D \cdot \alpha}\right)
$$

Good temporal correlation requires that our filter bandwidth be approximately one-half this, or

$$
\Delta \lambda=\bar{\lambda}^{2}\left(\frac{1}{2 \pi \cdot D \cdot \alpha}\right)
$$


The author acknowledges insights provided by Dr Hans Nieuwenhuijzen which assisted in this derivation. Another derivation of this appears in reference 4 .

\section{SOLAR LIMB EXPERIMENT}

The sun is very bright. It is a logical source to use for experiments involving narrow band filters and low sensitivity detectors. The sun's limb in the light of Hydrogen Alpha shows spicules of high spatial frequency content. An unsuccessful attempt was made to reconstruct a line image of the solar limb. However, a measurement of the "time constant" for atmospheric fluctuations was made. A brief description of the experiment and the results is given here.

Theory indicates that diffraction limit information is obtained by a numerical analysis of the intensity fluctuations at the image of the pupil plane as relayed through the 180 -degree rotationally shearing interferometer ${ }^{3,4}$. In particular the information lies in the variance of the intensity fluctuations. Intensity fluctuations at the pupil as relayed through the interferometer were recorded with 256 channels of a Reticon linear photodetector array.

We used the $60 \mathrm{~cm}$ vacuum telescope of the Kitt Peak National Observatory in conjunction with the 512 channel linear "Reticon" detector array, and Varian $620 \mathrm{~F}$ computer system ${ }^{11}$ to examine intensity fluctuations at the pupil plane. The image plane irradiance distribution was the extreme solar limb, masked by an aperture of 6.7 arc-seconds.

The telescope has an aperture diameter of $60 \mathrm{~cm}$, with the center $16.5 \mathrm{~cm}$ diameter blocked. The focal length of the image-forming mirror is 31 meters, which yields a scale of 6.7 arc-seconds per millimeter at the image plane.

We positioned the instrument horizontally over the spectrograph of the vacuum telescope to intercept light converging to the entrance slit. The interferometer output (an image of the telescope pupil) is relayed onto the central 256 channels of the 512 channel Reticon array We sample the $60 \mathrm{~cm}$ aperture with approximately 210 elements of this array, each having size 25 by 250 microns. 
Figure 3 shows a schematic view of the intensity as a function of position across the secondary, or "double" image plane for the case of the solar limb across the circular aperture at the image plane. Distance $\mathrm{r}$, is approximately 0.1 millimeter.

The sampling spatial frequency was therefore 3.5 sensitive elements per centimeter at the pupil. The irradiance at each element was integrated for 1/60 second and sampled sequentially at $60 \mathrm{~Hz}$. Each integrated intensity was converted to 8 bits of digital information and written onto a magnetic tape.

Aperture positioned at the image plane limited the diameter of the area processed to $1.0 \mathrm{~mm}$ ( 6.7 arc-seconds or 5000 kilometers at the sun). Several sets of observations were taken with the solar limb approximately bisecting the aperture. Prior to each observing run the image plane aperture was carefully positioned so that the irradiance distribution at the secondary or "double" image plane resembled that shown in Figure 3. This figure shows the orientation of the limb. The long direction of the diode array is oriented parallel to the line connecting the centers of the images of the apertures

The number of spatial elements required for sampling at the Nyquist rate across the pupil is 318 . The available apparatus gave 210 samples. Consequently, it was necessary to sample with considerable ailaising.

We recorded data through one of two interference filters. One f1lter has a 5 nanometer bandpass at $\mathrm{H} \alpha(656 \mathrm{~nm})$, the other has a 10 nanometer bandpass at $510 \mathrm{~nm}$.

A typical observation time required about 60 seconds, during which time al1 256 diode array elements are sampled at the rate of $60 \mathrm{~Hz}$. Data were stored sequentially on magnetic tape in approximately 3,600 blocks, each having a length of 256 data points Each data point consists of one-half of a Varian 620L 16-bit word. The bit transfer rate of approximately $480 \mathrm{~Hz}$ was limited by the speed of the magnetic tape unit. Data were written directly on tape as fast as they were taken because no intermediate high-speed storage device such as a disk was available on this system ${ }^{11}$. 
$31-8$
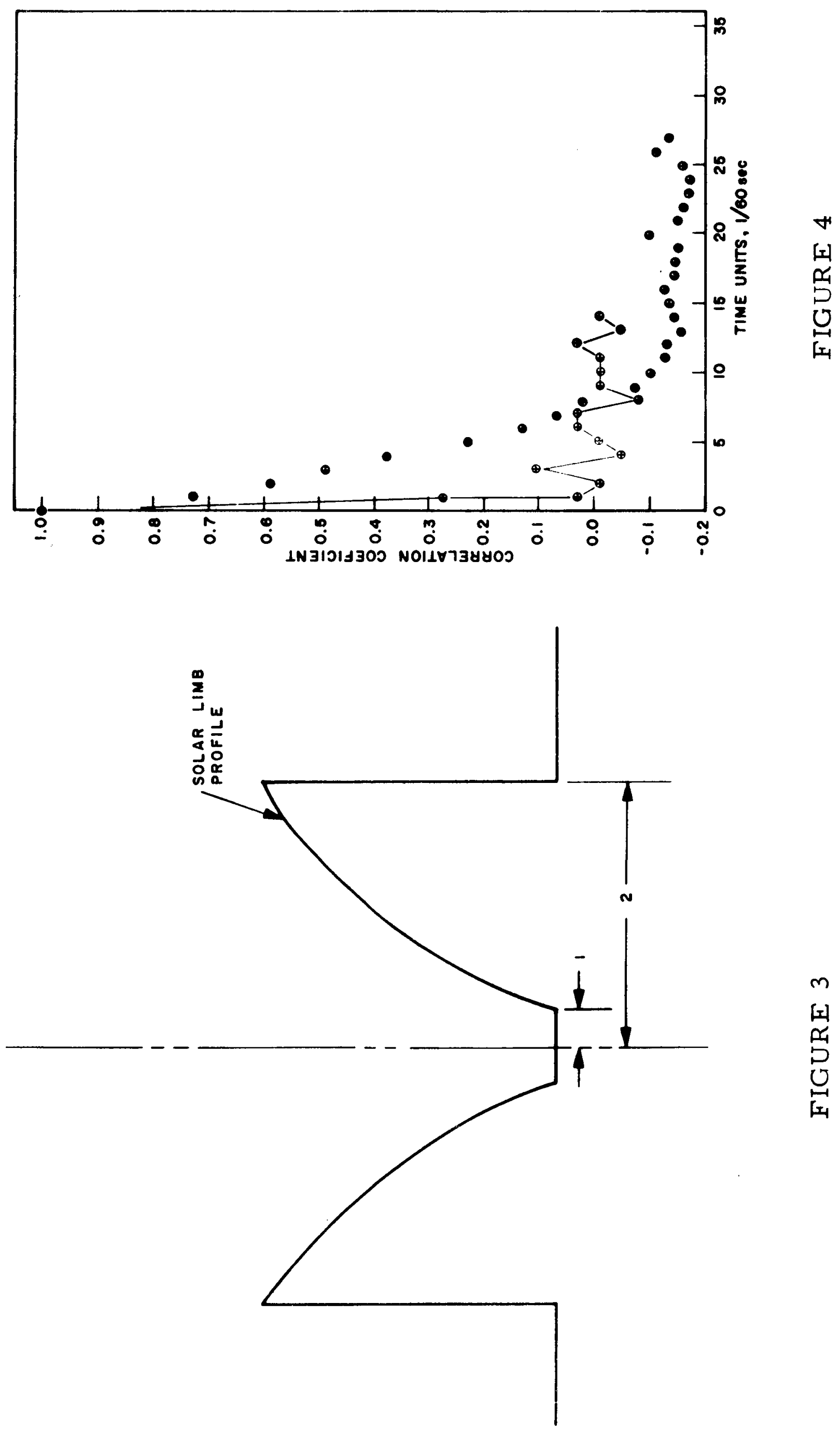

孚 
The Varian 620 utilizes a 16-bit word. A routine for unpacking each of the two eight-bit data words within the one 16-bit Varian word and converting the data tapes to the 60-bit word format of the CDC 6400 was provided by the Kitt Peak Computer Center. Corrections for zero offset and gain variations among the sensitive elements of the array, as well as calculations of variance, envelope, and power spectra were all made by the author using FORTRAN VI with the KPNO CDC 6400.

Several sets of data on the solar limb were taken on November 22 and 23, 1975. We made 4 runs with 1.0 millimeter aperture (6.7 arc-seconds) We corrected for zero offset and gain differences between sensitive elements using data recorded with no illumination on the diodes and with nominally unfform 1llumination, assuming that the diodes have a response that is linear with irradiance. The data were reduced by processing 1,408 1/60-second samples for each of the 256 spatial samples.

We now give a quantitative analysis of the data reduction technique. The silicon photodiodes have a voltage response at the ith element at time $t$ given by

$$
V_{i}(t)=I_{i}(t) \cdot g_{1}+V_{o i}
$$

where $I_{i}(t)$ is the intensity of the light falling on diode element $i$ at time $t$ which has gain $g_{i}$ and voltage offset $v_{o i}$

The zero offset $V_{o 1}$ is given by a data run with $I=0$, so that

$$
v_{1}(t) \text { dark }=V_{o i}
$$

(equation 4)

The gain of each diode element is calculated from the same data we use for the calculation of variance and envelope. The intensities are averaged in time. We assume that this average closely approximates the signal which would be obtained if we 1lluminated the diodes uniformly That is, we assume that the average irradiance level is the same for all 256 elements It is not necessary to know what it is. We calibrate the diodes with the identical 
optical system we use for the data acquisition. The effects of sma11 variations in the transmissivity across the diode array are cancelled. With these conditions, and Equation 3 and Equation 4 become

$$
V=V_{i}(t)=\left[I_{i}\right]_{t} \cdot g_{i}+V_{i}(t) \text { dark (equation 5) }
$$

where the bracket refers to a time average. We assume that neither the gain of each sensitive element nor its zero offset is time-dependent.

We assume that for uniform illumination, $V$ is normalized to 100 , and calculate the relative gain of each diode from Equation 3:

$$
g_{i}=\frac{100-v_{i}(t)}{\left[I_{i}\right]_{t}}
$$

We use the relationships given in Equation 3 with constants calculated with (4) and (6) and to correct all the data.

We first examine the data to determine if the time interval between our samples was sufficiently long to permit independent samples, but yet sufficiently short to "freeze" fringe motion induced by atmospheric turbulence. The autocorrelation coefficient in time is defined as

$$
C(T, x)=\frac{1}{2 T} \int_{-T}^{T} I(t-T, x) \cdot I(t, x) d t \quad \text { (equation 7) }
$$

Where $I(t)$ is the instantaneous intensity recorded at a particular sensitive element at time $t$.

We calculated the time autocorrelation coefficient $\mathrm{C}(\mathrm{T}, \mathrm{x})$ for the data runs. We chose sample point number 97 (out of 256) for this calculation. This point sampled the intensity fluctuations introduced by the atmosphere on the signal at the pupil. 
Figure 4 shows a graph of this time autocorrelation function for a dark run, calculated using 256 time samples. We see that the normalized autocorrelation coefficient drops to 0.03 in the time of $1 / 60$ second and remains below 0.1 . Fluctuations which appear beyond $3 / 60$ second are probably noise. Figure 4 also shows a plot of this autocorrelation coefficient as a function of time in 1/60 second time intervals for solar data run. This correlation coefficient drops to 0.5 in $3 / 60$ second. It is safe, therefore, to assume that the electronic circuits are sufficiently fast to follow atmospheric fluctuations and that correlations we observe with a frame time of $1 / 60$ second do not originate with the response of the detector system. We conclude that the data plotted in Figure 4 show the correlation in time of fluctuations in the Earth's atmosphere.

The method for recording data integrates the signal over a time period of $1 / 60$ second. From data shown in Figure 4 we see that during the $1 / 60$ second integration the signal we wish to observe, that is, the fringe amplitude becomes decorrelated to a correlation coefficient of 0.7 . Consequently, the observed fringe contrast as calculated from the observed intensity fluctuations is reduced. The signal-to-noise of our data would be improved if this integration time could be reduced by a factor of 2 . The length of time between samples should be increased by a factor of two.

A better system would be to mount an electro-optic shutter in front of the array and sequence the data acquisition as follows: open shutter for $1 / 120$ second, so that all diode sensitive elements charge simultaneously, then during a dark interval of $1 / 30$ second the diode charge is read out by the electronic circuitry, converted through the analog to digital converter, data words are packed by the software and then written onto magnetic tape. This sequence of $1 / 120$ second for observing with all diodes, followed by $1 / 30$ second to let the atmosphere settle prior to the next observation would improve the signal-tonoise ratio at each sample, and permit more diode elements to sample the pupil intensity fluctuations.

The autocorrelation function in time was computed for several spatial sampling points other than Number 97 and found to have the same characteristics as those shown in Figure 4. 
The behavior of the spatial autocorrelation coefficient shows that the electronic circuitry for reading the charge on the diodes does not introduce artificial spatial correlations.

In other papers we have shown that the signal information of interest lies in the variance of the intensity fluctuations at each point or the pupil ${ }^{3,4}$.

This preliminary experiment, although not resulting in a line image of the solar limb was considered partially successful because of knowledge obtained about the atmosphere and experience with the CCD array

\section{THE FUTURE}

A two-dimensional array detector, examining intensity fluctuations in the Michelson stellar interferometer fringe pattern from an extended source may lead to passive image reconstruction at diffraction limit of large telescopes. Advances in computational techniques appear to be helpful 12,13 .

\section{ACKNOWLEDGEMENT}

The author wishes to thank Drs John 0. Stoner, Rolland Shack, and James Burke of the Optical Sciences Center for their guidance during my efforts on this dissertation work. Drs. K. Pierce and J.W. Brault of Kitt Peak National Observatory were most helpful.

This paper presents the results of one phase of research carried out at the Jet Propulsion Laboratory, California Institute of Technology, under Contract Number NAS 7-100, sponsored by the National Aeronautics and Space Administration (and under Contract DOT-AS-20094, sponsored by the C1imatic Impact Assessment Program of the Department of Transportation). 


\section{REFERENCES}

1) James B. Breckinridge, "Coherence Interferometer and Astronomical Applications," Applied Optics, 11, 2996-2998, 1972.

2) James B. Breckinridge, "Two-Dimensional White Light Coherence Interferometer," Applied Optics, 13, 2760-2762, 1974.

3) James B. Breckinridge, "The Spatial Structure Analyzer and Its Astronomical Applications," PhD, Optical Sciences Dissertation, University of Arizona, 1976.

4) James J Burke and James B. Breckinridge, "Passive Imaging Through the Turbulent Atmosphere: Fundamental Limits on the Spatial Frequency Resolution of a Rotational Shearing Interferometer," J. Opt. Soc. Amer, 68, 67-77, 1978 .

5) C. Roddier, "Measurements of the Atmospheric Attenuation of the Spectra1 Components of Astronomical Images," J. Opt. Soc. Amer., 66, 478-482.

6) J.B. Breckinridge, "Measurement of the Amplitude of Phase Excursions in the Earth's Atmosphere," J. Opt. Soc. Amer., 66, 143-144.

7) J.B. Breckinridge, "Interference in Astronomical Speckle Patterns," J. Opt Soc. Amer., 66, 1240-1242, 1976.

8) J.B. Breckinridge, H.A. McAlister and W.G. Robinson, "The Kitt Peak Speckle Camera," submitted to Applied Optics.

9) D.W. Cutter and A.W. Lohmann, "Achromatic Stellar Interferometry," Optics Communications, 12, 220-222, 1974.

10) G.R. Wlock, Optics Communications, 18, 307, 1976.

11) W.C. Livingston, J Harvey, C. Slaughter, and D. Trumbo, Applied Optics, $15,40-51,1976$.

12) A.H. Greenaway, "Proposal for Phase Recovery from a Single Intensity Distribution," Optics Letters, 1, 10-12, 1977.

13) J.R. Fienup, "Reconstruction of an Object From the Modulus of its Fourier Transform," Optics Letters, $\underline{3}, 27-30,1978$. 